\section{EFFICIENT ANALYSIS OF LARGE PHASED ARRAYS USING ITERATIVE MOM WITH DFT-BASED ACCELERATION ALGORITHM}

\author{
V. B. Ertürk ${ }^{1}$ and Hsi-Tseng Chou $^{2}$ \\ ${ }^{1}$ Department of Electrical and Electronics Engineering \\ Bilkent University \\ TR-06800, Bilkent \\ Ankara, Turkey \\ 2 Department of Electrical Engineering \\ Yuan Ze University \\ 135 Yuan-Tung Rd. \\ Chung-Li 320, Taiwan
}

Received 27 March 2003

\begin{abstract}
A discrete Fourier transform (DFT)-based iterative method of moments (IMoM) algorithm is developed to provide an $O\left(N_{\text {tot }}\right)$ computational complexity and memory storages for the efficient analysis of electromagnetic radiation/scattering from large phased arrays. Here, $N_{\text {tot }}$ is the total number of unknowns. Numerical results for both printed and free-standing dipole arrays are presented to validate the algorithm's efficiency and accuracy. (C) 2003 Wiley Periodicals, Inc. Microwave Opt Technol Lett 39: 89-94, 2003; Published online in Wiley InterScience (www.interscience.wiley.com). DOI 10.1002/mop. 11136
\end{abstract}

Key words: phased array; method of moments; discrete Fourier transform; iterative solvers

\section{INTRODUCTION}

There is a growing interest in the analysis of the electromagnetic radiation/scattering from large and finite phased arrays, due to the fact that present and future EM sensor systems are expected to utilize large phased arrays. However, when the number of elements in the array increases, majority of the conventional analysis methods suffer greatly from the memory storage requirements and computing time. The conventional method of moments (MoM) [1] requires an operational count of $O\left(N_{\text {tot }}^{3}\right)$ (of order $N_{\text {tot }}^{3}$ ) and memory storage of $O\left(N_{t o t}^{2}\right)$, where $N_{t o t}$ is the total number of elements, whereas both the memory storage and the operation count for MoM in conjunction with iterative schemes (iterative $\mathrm{MoM})$ are $O\left(N_{t o t}^{2}\right)$ for ordinary matrix-vector multiplication at each iteration. Many methods have been attempted to simplify the computational complexity and to reduce the memory requirements [2-12]. Among them, the fast multipole method (FMM) [2] (with operational count $O\left(N_{t o t}^{1.5}\right)$ and its subsequent extensions [3, 4], multi-level FMM with operational count $O\left(N_{\text {tot }} \log N_{\text {tot }}\right.$, as well as conjugate gradient-fast Fourier transform (CG-FFT with $O\left(N_{\text {tot }^{-}}\right.$ $\log N_{t o t}$ ) [5], are some typical successful efforts. Other examples of analysis methods to reduce the number of unknowns in MoM modeling, based on a hybrid combination of MoM with either uniform geometrical theory of diffraction (UTD) [11] or discrete Fourier transform (DFT) [12], can also be found in the literature. On the other hand, several infinite array approaches to handle large finite arrays exist in the literature [13]. However, none of them provides the complete finite array truncation effects, which significantly effects both the element input impedance and the array radiation/scattering patterns.

In this paper, a discrete Fourier transform (DFT) [14] based acceleration algorithm [7] is used in conjunction with the biconjugate gradient stabilized method (Bi-CGSTABM) to reduce the computational complexity and memory storage of the iterative MoM (IMoM) solution to $O\left(N_{t o t}\right)$ in the analysis of phased arrays of both free-standing and printed dipoles. The $O\left(N_{t o t}\right)$ complexity of the solution is due to this DFT-based acceleration algorithm, which divides the contributing elements into "strong" and "weak" interaction groups for a receiving element in the IMoM. The contributions from the strong group are obtained by conventional element-by-element computation to assure fundamental accuracy. On the other hand, the entire induced current distribution is expressed in terms of a global domain DFT representation. Significant DFT terms are identified based on a previous work on DFTMoM [12] and employed to obtain the interactions from the weak group. In general, only a few significant DFT terms are sufficient to provide accurate results due to the fact that they provide minor corrections to the solution in contrast to the dominating strong group.

It should be noted at this point that, recently, the same DFTbased acceleration technique was used in conjunction with a forward-backward method (FBM) to analyze large phased arrays of free-standing dipoles in [7] and then extended to large phased arrays of printed dipoles in $[8,9]$. The use of FBM in conjunction with this DFT-based acceleration algorithm shows some significant convergence problems in the analysis of printed dipole phased arrays when either the thickness of the substrate or the relative dielectric constant of the substrate $\left(\epsilon_{r}>1\right)$ (or both) are high [8, 9]. However, such problems have not been observed in [10] nor in this study.

This paper is organized in the following order. In section 2, the IMoM formulation of the problem for a large phased array of both free-standing and printed dipoles is reviewed, together with a brief explanation of Bi-CGSTABM. Section 3 presents the DFT-based acceleration algorithm. In section 4 , numerical results are presented using this method and compared with conventional MoM to validate the method's efficiency and accuracy. An $e^{j \omega t}$ time dependence is assumed and suppressed throughout this paper.

\section{REVIEW OF IMOM AND BI-CGSTABM}

Consider a uniformly excited, rectangular, finite, planar, periodic array of $(2 N+1) \times(2 M+1)$ dipoles. The array elements might be identical, short and thin, perfectly conducting wire dipole elements oriented along the $x$ direction in the $z=0$ plane in air (free-standing dipoles), as illustrated in Figure 1(a), or identical printed dipoles on a grounded dielectric substrate with a thickness $d$ and relative dielectric constant $\epsilon_{r}$, as depicted in Figure 1(b). For both geometries, each dipole is assumed to have a length $L$ and width $W$, and is uniformly spaced from its neighbors by distances $d_{x}$ and $d_{y}$ in the $x$ and $y$ directions, respectively. The dipoles are assumed to be center fed with infinitesimal generators.

\subsection{The Method of Moments Solution}

The dipoles are assumed to be thin $(W \ll L$ ), so that only $\hat{x}$-directed currents are required. Therefore, the current distribution on each dipole is given by

$$
I_{n m}\left(x^{\prime}\right)=A_{n m} P_{n m}\left(x^{\prime}\right) f_{n m}\left(x^{\prime}\right),
$$

where $A_{n m}$ is the unknown coefficient of $f_{n m}\left(x^{\prime}\right)$ that determines the current at the feed point on the element, and

$$
P_{n m}(x)= \begin{cases}1 & x \in n m^{\text {th }} \text { element } \\ 0 & \text { otherwise. }\end{cases}
$$

For the free-standing dipole array, the current $f_{n m}\left(x^{\prime}\right)$ on the $n m^{\text {th }}$ $(-N \leq n \leq N,-M \leq m \leq M)$ dipole is of the type 


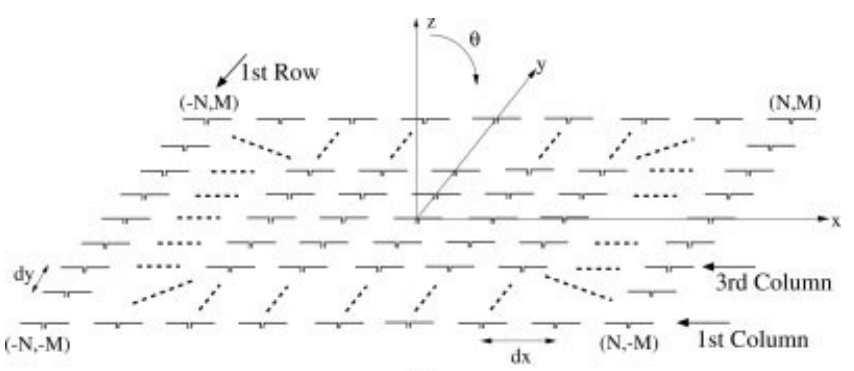

(a)

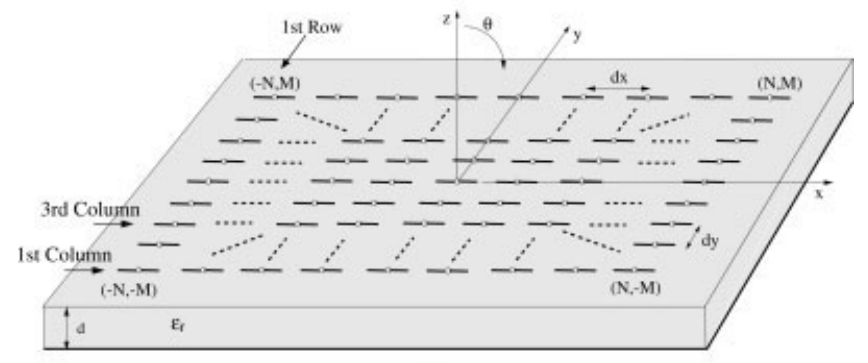

(b)

Figure 1 Geometry of a periodic array of $(2 N+1) \times(2 M+1)($ a $)$ free-standing dipoles and (b) printed dipoles

$$
f_{n m}\left(x^{\prime}\right)=\sin \left[k_{0}\left(\frac{L}{2}-\left|x^{\prime}-n d_{x}\right|\right)\right]
$$

where $k_{0}$ is the free space wave number. Similarly, for the printed dipole array, the current $f_{n m}\left(x^{\prime}\right)$ on the $n m^{\text {th }}$ dipole is expanded in terms of piecewise sinusoidal (PWS) modes, defined as

$$
f_{n m}\left(x^{\prime}\right)=\frac{\sin \left[k_{a}\left(\frac{L}{2}-\left|x^{\prime}-n d_{x}\right|\right)\right]}{W \sin \left(k_{a} \frac{L}{2}\right)},
$$

where $k_{a}=k_{0} \sqrt{\left(\epsilon_{r}+1\right) / 2}$ is the wavenumber of the expansion mode.

An electric field integral equation (EFIE) is formed by enforcing the boundary condition, such that the total $E_{x}$ field must vanish on the dipole surfaces. This EFIE is solved using a Galerkin MoM solution, leading to the following set of linear equations:

$$
\overline{\mathbf{Z}} \cdot \mathbf{I}=\mathbf{V}
$$

where $\overline{\mathbf{Z}}=\left[Z_{n m, p q}\right], \mathbf{I}=\left[A_{n m}\right]$, and $\mathbf{V}=\left[V_{p q} e^{-j k_{x} p d_{x}} e^{-j k_{y} q d_{y}}\right]$. In Eq. (5), the right-hand side is related to the excitation at the $p q^{\text {th }}$ dipole to radiate a direction beam according to $\left(k_{x}, k_{y}\right)$, defined by

$$
k_{x}=k_{0} \sin \theta_{i} \cos \phi_{i} ; k_{y}=k_{0} \sin \theta_{i} \sin \phi_{i}
$$

where $\left(\theta_{i}, \phi_{i}\right)$ is the scan direction of the beam. Also, $Z_{n m, p q}$ on the left-hand side is the mutual impedance between the $n m^{\text {th }}$ and $p q^{\text {th }}$ dipoles, and is given by

$$
Z_{n m, p q}=j \omega \mu \int_{L_{p q}} d x \int_{L_{n m}} d x^{\prime} f_{p q}(x) G_{x x}\left(\mathbf{r}_{p q} \mid \mathbf{r}_{n m}^{\prime}\right) f_{n m}\left(x^{\prime}\right)
$$

where $\mathbf{r}_{p q}$ and $\mathbf{r}_{n m}^{\prime}$ are the position vectors of the $p q^{\text {th }}$ and $n m^{\text {th }}$ dipoles and $G_{x x}\left(\mathbf{r}_{p q} \mid \mathbf{r}_{n m}^{\prime}\right)$ is the corresponding component of the (i) free-space dyadic Green's function for the free-standing dipole array, and the (ii) planar microstrip dyadic Green's function [16] for the printed dipole array.

Finally, note that for a scattering problem where an external plane wave is incident on the array, in which the elements are now passive, the right-hand side of Eq. (5) is associated with the incident field $E_{x}^{i}\left(\mathbf{r}_{p q}\right)$, namely,

$$
E_{x}^{i}\left(\mathbf{r}_{p q}\right)=V_{p q} e^{-j k_{x} p d_{x}} e^{-j k_{y} q d_{y}} \delta\left(x-p d_{x}\right) .
$$

\subsection{BiConjugate Gradient Stabilized Method (Bi-CGSTABM)}

Bi-CGSTABM is a very reliable form of the conjugate gradient method (CGM) in terms of convergence. In this method, let $\mathbf{I}_{i}$ be the value of $\mathbf{I}$ at the $i^{\text {th }}$ iteration with $\mathbf{I}_{0}$ the initial guess. Then,

$$
\mathbf{R}_{0}=\mathbf{V}-\overline{\mathbf{Z}} \cdot \mathbf{I}_{0}, \mathbf{S}_{0}=\mathbf{R}_{0}
$$

At the $i^{\text {th }}$ iteration, the Bi-CGSTABM updates each vector in the following way:

$$
\alpha_{i}=\frac{\left\langle\mathbf{R}_{i}, \mathbf{R}_{i}\right\rangle}{\left\langle\overline{\mathbf{Z}} \cdot \mathbf{S}_{i}, \mathbf{S}_{i}\right\rangle}, \mathbf{I}_{i+1}=\mathbf{I}_{i}+\alpha_{i} \mathbf{S}_{i}, \mathbf{R}_{i+1}=\mathbf{R}_{i}-\alpha_{i} \overline{\mathbf{Z}} \cdot \mathbf{S}_{i}
$$

and

$$
c_{i}=\frac{\left\langle\mathbf{R}_{i+1}, \mathbf{R}_{i+1}\right\rangle}{\left\langle\mathbf{R}_{i}, \mathbf{R}_{i}\right\rangle}, \mathbf{S}_{i+1}=\mathbf{R}_{i+1}+c_{i} \mathbf{S}_{i},
$$

where $\langle$,$\rangle denotes the inner product without complex conjugate.$ This procedure continues until a converged result is obtained. Details of this method can be found in [15].

\section{ACCELERATION ALGORITHM BASED ON DFT EXPANSION}

The repeated and time-consuming computations of $\overline{\mathbf{Z}} \cdot \mathbf{I}\left[\mathbf{I}=\mathbf{I}_{0}\right.$ in Eq. (9) and $\mathbf{I}=\mathbf{S}_{i}$ in Eq. (10)] type matrix-vector multiplications in IMoM are accelerated using this DFT-based acceleration algorithm. In this algorithm, the contributing elements are divided into "strong" and "weak" interaction groups, as depicted in Figure 2(a), such that

$$
\overline{\mathbf{Z}} \cdot \mathbf{I}=\sum_{n m \in \text { strong }} A_{n m} Z_{n m, p q}+\sum_{n m \in \text { weak }} A_{n m} Z_{n m, p q} .
$$

The contributions coming from the strong interaction group are obtained via exact element-by-element computation and are the same for each receiving element. The size of the strong region is fixed and very small, compared to the entire array. Therefore, the computational complexity remains $O\left(N_{t o t}\right)$. Since the dominating contributions are, in general, radiated from the strong group, the exact computation assures the fundamental accuracy of the method. On the other hand, the computation of contributions coming from the weak interaction group is based on a DFT expansion of the entire induced current on the array. Consequently, the contributions coming from the weak region to the $p q^{\text {th }}$ element is given by

$$
E_{\text {weak }}\left(\mathbf{r}_{p q}\right)=\sum_{n m \in \text { weak }} A_{n m} Z_{n m, p q}
$$




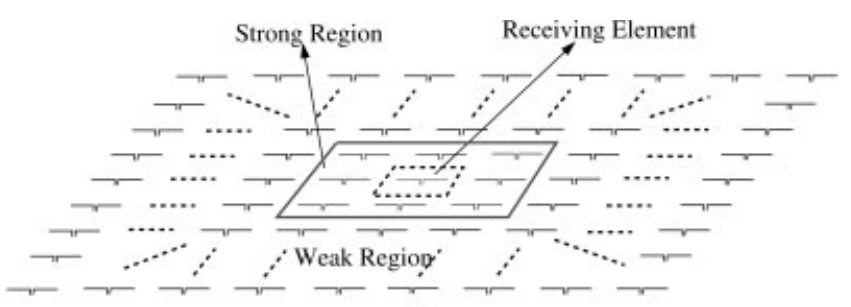

(a)

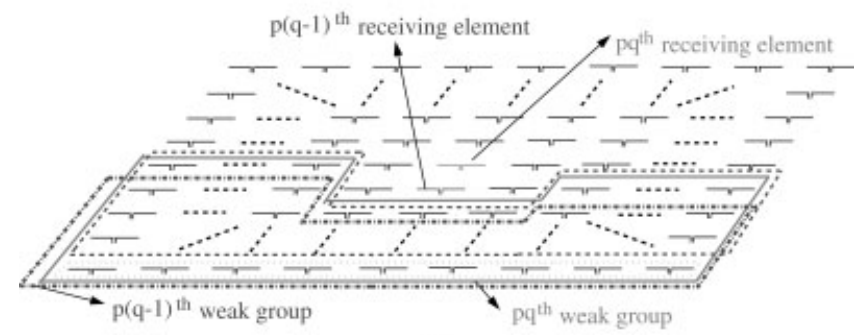

(b)

Figure 2 (a) Decomposition of interaction elements in terms of strong and weak groups; (b) the forward weak group corresponding to the $p q^{\text {th }}$ receiving element (the solid loop) is decomposed into two groups indicated by dotted and dashed loops. The dashed loop is identical to the dash-dotted loop, which is the forward weak group of $p(q-1)^{\text {th }}$ receiving element, except for a location shift, corresponding to a phase shift

and by substituting the DFT representation [14] of $A_{n m}$ into Eq. (13), we obtain

$$
\begin{aligned}
& E_{\text {weak }}\left(\mathbf{r}_{p q}\right)=\sum_{k=-N}^{N} \sum_{l=-M}^{M} B_{k l} \\
& \quad \times \sum_{n m \in \text { weak }} Z_{n m, p q} e^{-j k_{x} n d_{x}} e^{-j k_{y} m d_{y}} e^{-j 2 \pi(k n / 2 N+1)} e^{-j 2 \pi(l m / 2 M+1)}
\end{aligned}
$$

where $B_{k l}$ is the coefficient of the $k l^{\text {th }}$ DFT term and can be found from the inverse DFT [14]. For a rectangular array, the significant DFT terms can be determined based on the criterion presented in [12], where the DFT terms of the orthogonal column $(k=0)$ and row $(l=0)$ dominate. However, considering the fact that contributions coming from the weak region provide minor corrections to the solution in contrast to the dominating strong group, very few significant DFT terms can be retained during the calculation of Eq. (14). Consequently, substituting (14) into (12) and keeping only the significant DFT terms, results in

$$
\overline{\mathbf{Z}} \cdot \mathbf{I}=\sum_{n m \in \text { strong }} A_{n m} Z_{n m, p q}+\sum_{k l \in Q} B_{k l} C_{k l, p q},
$$

where $Q$ denotes the selected DFT terms, and

$$
C_{k l, p q}=\sum_{n m \in \text { weak }} Z_{n m, p q} e^{-j k_{x} n d_{x}} e^{-j k_{y} m d_{y}} e^{-j 2 \pi(k n / 2 N+1)} e^{-j 2 \pi(l m / 2 M+1)}
$$

With this expression, only $Q$ DFT terms ( $Q$ is a small, fixed number), which can be computed and stored before the Bi-CGSTABM proceeds, need to be computed for a given receiving element in addition to the contributions from the strong group. Consequently, $O\left(N_{t o t}\right)$ of computational complexity can be obtained. Note that the coefficients of the DFT terms are initially obtained from the infinite array assumption, where only the central element $B_{00}$ is nonzero. Then, they are continuously updated at each iteration.

$C_{k l, p q}$ in Eq. (16) denotes the contribution of the $k l^{\text {th }}$ DFT term to the $p q^{\text {th }}$ receiving element, where each DFT term represents a linear phase impression. One way of fast computing $C_{k l, p q}$ is to employ asymptotic techniques [12], which will decompose Eq. (16) in terms of few ray-like contributions due to edge and corner diffractions. However, in this paper, an alternative approach is employed which retains the computation complexity at $O\left(N_{t o t}\right)$. In this approach, the weak group is also divided into "forward" and "backward" subgroups, where the "forward" group consists of elements in the front of the $p q^{\text {th }}$ receiving element, and the rest of the weak group elements are included in the "backward" group. It should be mentioned that the number of elements in these two groups varies according to the location of the $p q^{\text {th }}$ element, but the total number remains roughly the same for each receiving element.

It is assumed that the iterative procedure sweeps elements in the order of $-N \leq p \leq N,-M \leq q \leq M$ (that is, $p q=\{11$, $21,31, \ldots, 12,22, \ldots, 13,23, \ldots\})$. Then, one may find the contributions due to the "forward" subgroup $C_{k l, p q}^{f}$ and the "backward" subgroup $C_{k l, p q}^{b}$ independently but iteratively. $C_{k l, p q}$ is only their superposition. One may explain $C_{k l, p q}^{f}$ in the following form:

$$
\begin{aligned}
C_{k l, p q}^{f}= & \sum_{m n \in W} Z_{n m, p q} e^{-j k_{x} n d_{x}} e^{-j k_{y} m d_{y}} e^{-j 2 \pi(k n / 2 N+1)} e^{-j 2 \pi(l m / 2 M+1)} \\
& +\sum_{n=-N}^{N} Z_{n(-M), p q} e^{-j k_{x} n d_{x}} e^{-j k_{y}(-M) d_{y}} e^{-j 2 \pi(k n / 2 N+1)} e^{-j 2 \pi[l(-M) / 2 M+1]},
\end{aligned}
$$

where $W$ is the "forward" when the $(-M)^{\text {th }}$ column [or $1^{\text {st }}$ column with respect to Fig. 1(a) or (b)] of the array is subtracted. Note that the mutual impedance $Z_{n m, p q}$ depends only on $(p-n)$ and $(q-$ $m$ ) for a periodic structure. As a result, the first term on the right-hand side of Eq. (17) can be related to $C_{k l, p(q-1)}^{f}$ by

$$
\begin{aligned}
C_{k l, p q}^{f}= & C_{k l, p(q-1)}^{f} \cdot e^{-j k_{3} d_{y}} e^{-j 2 \pi(l / 2 M+1)} \\
& +\sum_{n=-N}^{N} Z_{n(-M), p q} e^{-j k_{x} n d_{x}} e^{-j k_{y}(-M) d_{y}} e^{-j 2 \pi(k n / 2 N+1)} e^{-j 2 \pi[l(-M) / 2 M+1]} .
\end{aligned}
$$

The details are illustrated in Figure 2(b). Basically, the forward weak group corresponding to the $p q^{\text {th }}$ receiving element [the solid loop in Figure 2(b)] is decomposed into two groups, as indicated by dotted and dashed loops. The dashed loop is identical to the dash-dotted loop, which is the forward weak group of $p(q-1)^{\text {th }}$ receiving element, except a location shift which corresponds to a phase shift. It is noted that in the forward sweep $C_{k l, p(q-1)}^{f}$ is obtained before $C_{k l, p q}^{f}$ is interested. The next step is the computation of the second term in Eq. (18), namely,

$$
D_{k l, p q}^{f}=\sum_{n=-N}^{N} Z_{n(-M), p q} e^{-j k_{x} n d_{x}} e^{-j k_{y}(-M) d_{y}} e^{-j 2 \pi(k n / 2 N+1)} e^{-j 2 \pi[l(-M) / 2 M+1]},
$$

which contains the contributions coming from a one-dimensional (1D) array with the receiving element located far away from this array. Then, utilizing the dependence of $Z_{n m, p q}$ on $(p-n)$, Eq. (19) can be expressed as 


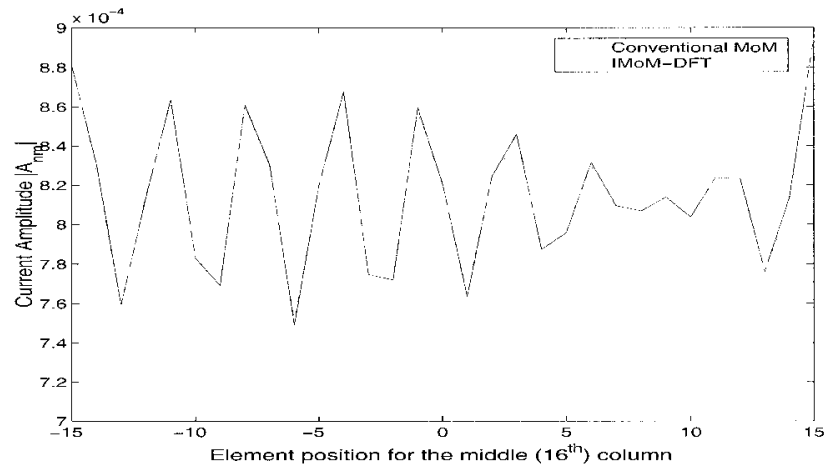

(a)

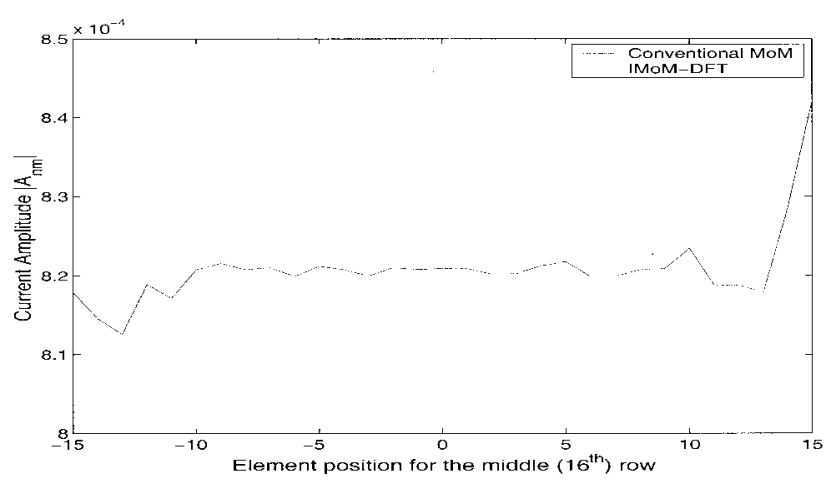

(b)

Figure 3 Comparison of the magnitude of induced current $\left|A_{n m}\right|$ obtained via IMoM-DFT and conventional MoM for the (a) middle $\left(16^{\text {th }}\right)$ column $(n=-15: 15$ and $m=0)$; (b) middle $\left(16^{\text {th }}\right)$ row $(m=$ $-15: 15$ and $n=0$ )

$$
\begin{aligned}
D_{k l, p q}^{f}= & \sum_{n=-N+1}^{N} Z_{n(-M), p q} e^{-j k_{x} n d_{x}} e^{-j k_{y}(-M) d_{y}} e^{-j 2 \pi(k n / 2 N+1)} e^{-j 2 \pi[l(-M) / 2 M+1]} \\
& +Z_{(-M)(-M), p q} e^{-j k_{x}(-N) d_{x}} e^{-j k_{y}(-M) d_{y}} \\
& \times e^{-j 2 \pi[k(-N) / 2 N+1]} e^{-j 2 \pi[l(-M) / 2 M+1]}
\end{aligned}
$$

and the first term can be related to $D_{k l,(p-1) q}^{f}$ by

$$
\begin{aligned}
D_{k l, p q}^{f} & =D_{k l,(p-1) q}^{f} \cdot e^{-j k_{x} d_{x}} e^{-j 2 \pi(k / 2 N+1)} \\
- & Z_{N(-M),(p-1) q} e^{-j k_{x}(N+1) d_{x}} e^{-j k_{y}(-M) d_{y}} e^{-j 2 \pi[k(N+1) / 2 N+1]} e^{-j 2 \pi[l(-M) / 2 M+1]} \\
& \quad+Z_{(-N)(-M), p q} e^{-j k_{x}(-N) d_{x}} e^{-j k_{y}(-M) d_{y}} e^{-j 2 \pi[k(-N) / 2 N+1]} e^{-j 2 \pi[(-M) / 2 M+1]} .
\end{aligned}
$$

Note that, if $q$ is in the first few columns where the strong region occupies all contributing rows, special treatments must be employed, even though the fundamental concepts are the same.

$C_{k l, p q}^{b}$, due to the "backward" group, can be found in a similar fashion by a backward sweep (that is, $p q=\{N M,(N-1) M$, $(N-2) M, \ldots, N(M-1),(N-1)(M-1), \ldots\})$. Indeed, the formulation is identical to (17)-(21), except that the multiplication "." is replaced by division " $/$ ".

The storage and complexity requirements of this algorithm are as follows: (a) storage of the unknown current vector in the iterative process is $N_{t o t}$; (b) storage of DFT terms is $Q$, where $Q$ $\ll N_{t o t}$ and is fixed regardless of the array size; (c) storage of $C_{k l, p q}$ is $Q \times N_{t o t}$; (d) the complexity of the strong region of computation is $N_{s} \times N_{t o t}$, where $N_{s} \ll N_{t o t}$ and $N_{s}$, the size of the strong region, is fixed regardless of the array size; (e) the complexity of the weak region of computation is $Q \times N_{t o t}$; (f) finally, the total complexity for the computations of $C_{k l, p q}$ and $D_{k l, p q}$ is $2 Q \times N_{t o t}$, consequently, the total cost is $O\left(N_{t o t}\right)$.

\section{NUMERICAL RESULTS}

In this section, the accuracy and efficiency of the proposed method are investigated. Numerical results for both free-standing and printed dipole arrays are obtained using this IMoM method with the DFT-based acceleration algorithm, and compared with the results obtained via conventional MoM. In all examples, the arrays are excited uniformly in amplitude so that $V_{p q}=1$ in Eq. (5) for each $p q^{\text {th }}$ dipoles.

In Figure 3(a) and (b), magnitude of the current distribution $\left(A_{n m}\right)$ of the middle column ( $16^{\text {th }}$ column, $\left.m=0\right)$ and middle row $\left(16^{\text {th }}\right.$ row, $\left.n=0\right)$ versus element position pertaining to a $31 \times 31$ free-standing dipole array with $d_{x}=0.6 \lambda_{0}$ and $d_{y}=0.3 \lambda_{0}$ (with $\lambda_{0}$ the free-space wavelength) are shown. The length and the radius of each dipole is $0.4 \lambda_{0}$ and $0.0005 \lambda_{0}$, respectively. The elements are phased to radiate a beam maximum in the direction of $(\theta=$ $\left.20^{\circ}, \phi=10^{\circ}\right)$. For this example, 15 DFT terms are selected $(Q=$ $15)$ and the size of the strong region is $3 \times 3\left(N_{s}=9\right)$ with the receiving element being located at the center of the strong region. The results obtained from IMoM-DFT agree well with the referenced results.

As for the printed dipoles, Figure 4(a) and (b) depicts the magnitude of the current distribution $\left(A_{n m}\right)$ of the middle $\left(21^{\text {st }}\right.$ column, $m=0$ ) and last ( $41^{\text {st }}$ column, $\left.m=20\right)$ columns versus

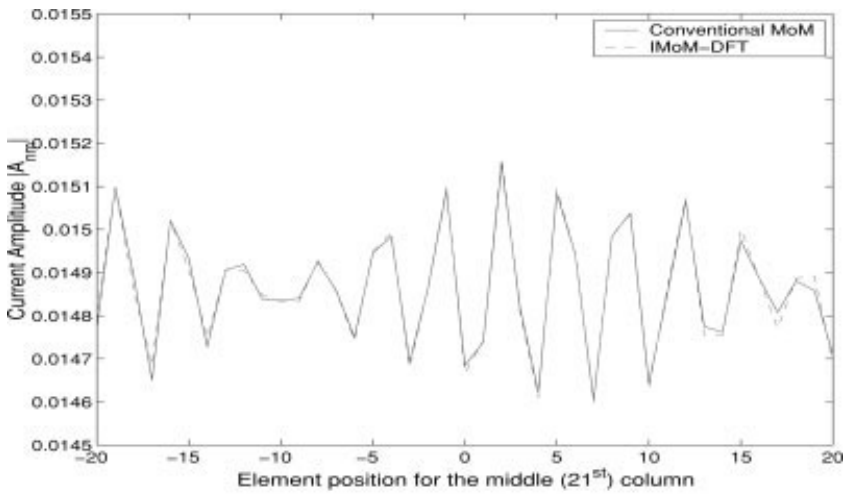

(a)

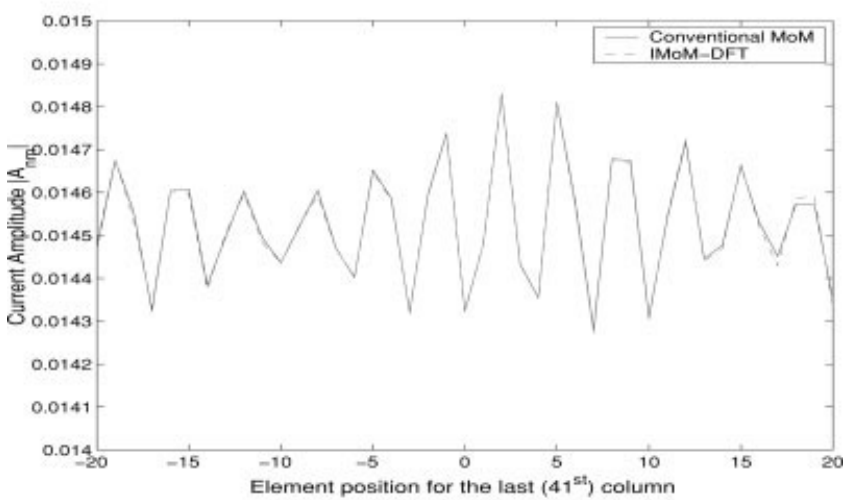

(b)

Figure 4 Comparison of the magnitude of induced current $\left|A_{n m}\right|$ obtained via IMoM-DFT and conventional MoM for the (a) middle $\left(21^{\text {st }}\right)$ column $(n=-20: 20$ and $m=0)$; (b) last $\left(41^{\text {st }}\right)$ column $(n=$ $-20: 20$ and $m=20$ ) 
element position, pertaining to a $41 \times 41$ printed dipole array with $d_{x}=d_{y}=0.5 \lambda_{0}$. The slab has a thickness of $0.05 \lambda_{0}$ (relatively thin) and its relative permittivity is $\epsilon_{r}=2.55$. The length and width of each dipole is $0.39 \lambda_{0}$ and $0.01 \lambda_{0}$, respectively, and the elements are phased to radiate a beam maximum in the direction of $\left(\theta=30^{\circ}, \phi=40^{\circ}\right)$. Overall, five DFT terms with a strong region of $3 \times 3$ are used. Very good agreement between IMoM-DFT and conventional MoM is obtained, as illustrated by these results.

In a similar way, a $31 \times 31$ printed dipole array with the same parameters for each element is investigated for a thicker substrate $d=0.19 \lambda_{0}$ with the same $\epsilon_{r}=2.55$. The comparison between the IMoM-DFT and conventional MoM of the magnitude of the current distribution $\left(A_{n m}\right)$ of the $2^{\text {nd }}(m=-14)$ and $13^{\text {th }}(m=$ $-3)$ are illustrated in Figure 5(a) and (b) for a broadside scan case $\left(\theta=0^{\circ}, \phi=0^{\circ}\right)$. Again, fairly good agreement is obtained. The only problem is that, regardless of the size of the array, as the thickness of the substrate increases due to the surface waves, more DFT terms (or a slight increase in the strong region) may be required. Therefore, in this example $Q=21$, although the strong region has still the same size $(3 \times 3)$. Nevertheless, this is a case where FBM accelerated with a DFT-based algorithm has convergence problems, which are probably due to the nature of FBM, as claimed in [9].

Finally, the radiation pattern $\left(E_{\theta}\right)$ of a $21 \times 21$ printed dipole array, with $d_{x}=d_{y}=0.5 \lambda_{0}, d=0.05 \lambda_{0}$, and $\epsilon_{r}=2.55$ is obtained with IMoM-DFT and compared with the result of the conventional MoM in Figure 6. The length and the width of each element is $l_{d i p}=0.39 \lambda_{0}$ and $w_{d i p}=0.01 \lambda_{0}$, respectively, and elements are phased to radiate a beam maximum in the direction of

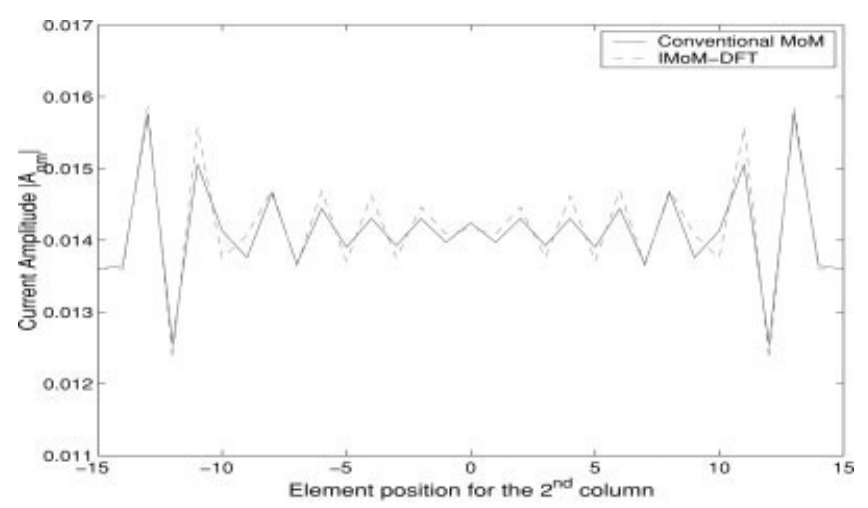

(a)

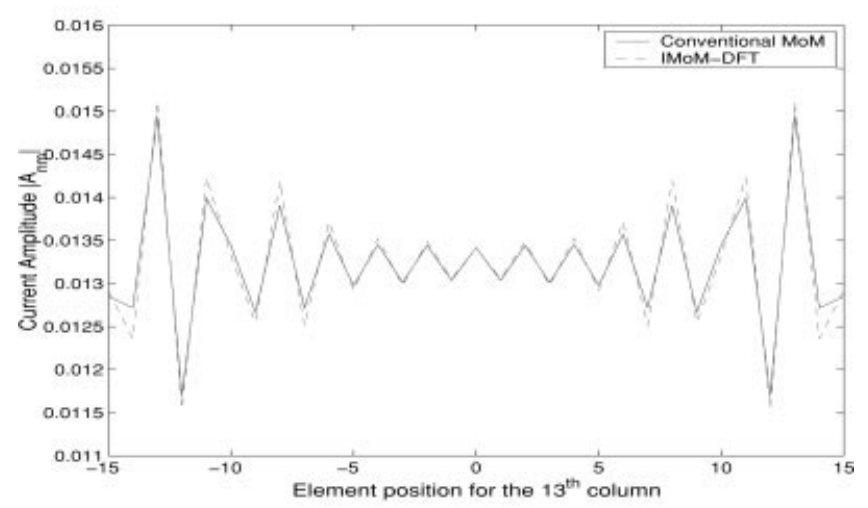

(b)

Figure 5 Comparison of the magnitude of induced current $\left|A_{n m}\right|$ obtained via IMoM-DFT and conventional MoM for the (a) $2^{\text {nd }}$ column ( $n=$ $-15: 15$ and $m=-14)$; (b) $13^{\text {th }}$ column $(n=-15: 15$ and $m=-3$ )

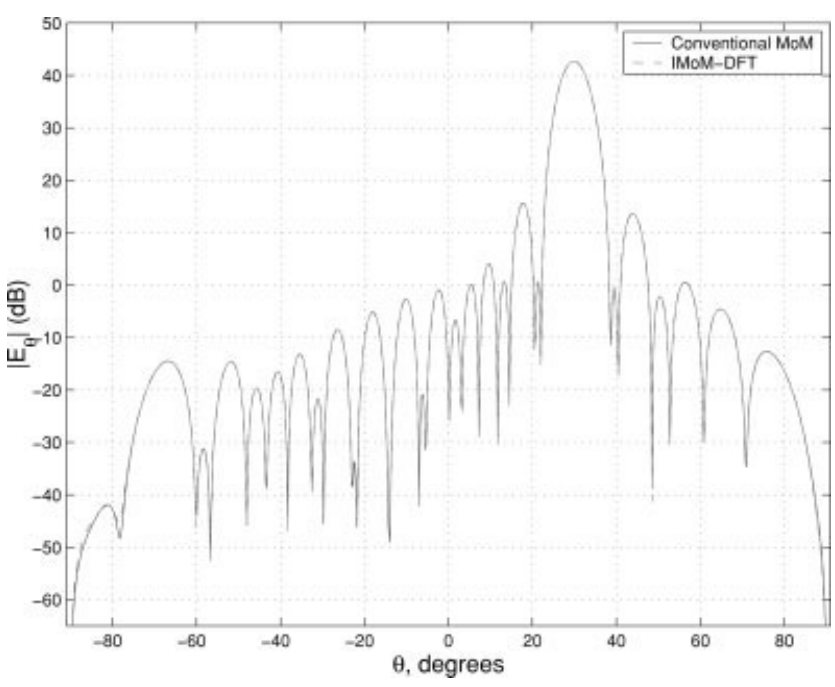

Figure 6 Far-field pattern of a $21 \times 21$ printed dipole array radiate a beam maximum in the direction of $\left(\theta=30^{\circ}, \phi=40^{\circ}\right)$

$\left(\theta=30^{\circ}, \phi=40^{\circ}\right)$. Agreement with the referenced result is again very good, thereby establishing confidence in the present IMoMDFT approach.

\section{DISCUSSION AND CONCLUSION}

An efficient and accurate analysis method for radiation/scattering from electrically large phased arrays of both free-standing and printed dipoles is presented. Both the computational complexity and the memory storage of the IMoM solution are reduced to $O\left(N_{t o t}\right)$ by using a DFT-based acceleration algorithm. Numerical results demonstrate the efficiency and accuracy of this method. The present approach will be extended to treat finite arrays with tapered excitations and non-rectangular array shapes. It should be noted that the contributions from DFT terms can also be efficiently found by asymptotic techniques [12], in terms of ray solutions. Consequently, they can be incorporated into ray-tracing codes to account for the interactions between the array and the environment.

\section{REFERENCES}

1. R.F. Harrington, Field computation by moment methods, IEEE Press, New York, 1993.

2. N. Engheta, W.D. Murphy, V. Rokhlin, and M.S. Vassiliou, The fast multipole method (FMM) for electromagnetic scattering problems, IEEE Trans Antennas Propagat 40 (1992), 634-641.

3. R.J. Burkholder and D.H. Kwon, High frequency asymptotic acceleration of the fast multipole method, Radio Sci 31 (1996), 1199-1206.

4. X.Q. Sheng, J.-M. Jin, J. Song, W.C. Chew, and C.-C. Lu, Solution of combined-field integral equation using multilevel fast multipole algorithm for scattering by homogeneous bodies, IEEE Trans Antennas Propagat 46 (1998), 1718-1726.

5. Y. Zhuang, K.-L. Wu, C. Wu, and J. Litva, A combined full-wave CG-FFT method for rigorous analysis of large microstrip antenna arrays, IEEE Trans Antennas Propagat 44 (1996), 102-109.

6. H.-T. Chou, Extension of the forward-backward method using spectral acceleration for the fast analysis of large array problems, IEE Proc Microwave Antennas Propagat 147 (2000), 167-172.

7. H.-T. Chou and H.-K. Ho, Implementation of a forward-backward procedure for the fast analysis of electromagnetic radiation/scattering from two dimensional large phased arrays, IEEE Trans Antennas Propagat (submitted).

8. Ö.A. Civi, Extension of forward backward method with DFT based acceleration algorithm for the efficient analysis of large printed dipole 
arrays, 2002 IEEE AP-S Int Symp Antennas Propagat and USNC/ URSI Natl Radio Sci Mtg, San Antonio, TX, 626-629.

9. Ö.A. Civi, Extension of forward-backward method with a DFT-based acceleration algorithm for efficient analysis of radiation/scattering from large finite-printed dipole arrays, Microwave Opt Technol Lett 37 (2003), 626-629.

10. V.B. Ertürk and H.T. Chou, Fast acceleration algorithm based on DFT expansion for the iterative MoM analysis of electromagnetic radiation/ scattering from two-dimensional large phased arrays, 2002 IEEE AP-S Int Symp Antennas Propagat and USNC/URSI Natl Radio Sci Mtg, San Antonio, TX, 156-159.

11. Ö.A. Civi, P.H. Pathak, H.-T. Chou, and P. Nepa, A hybrid uniform geometrical theory of diffraction-moment method for the efficient analysis of electromagnetic radiation/scattering from large finite planar arrays, Radio Sci 35 (2000), 607-620.

12. H.-T. Chou, H.-K. Ho, P.H. Pathak, P. Nepa, and Ö.A. Civi, Efficient hybrid discrete Fourier transform-moment method for fast analysis of large rectangular arrays, IEE Proc Microwave Antennas Propagat 149 (2002), 1-6.

13. A.K. Skrivervik and J.R. Mosig, Finite phased array of microstrip patch antennas: The infinite array approach, IEEE Trans Antennas Propagat 40 (1992), 579-582.

14. A.V. Oppenheim and R.W. Schafer, Discrete-time signal processing, Prentice Hall, New York, 1999.

15. T.K. Sarkar, On the applications of generalized biconjugate gradient method, J Electromagn Wave Appl 1 (1987), 223-242.

16. S. Barkeshli, P.H. Pathak, and M. Marin, An asymptotic closed-form microstrip surface Green's function for the efficient moment method analysis of mutual coupling in microstrip antennas, IEEE Trans Antennas Propagat 38 (1990), 1374-1383.

(C) 2003 Wiley Periodicals, Inc.

\section{LOSSY EFFECTS ON THE TRANSIENT PROPAGATION IN LTCC COPLANAR WAVEGUIDES (CPWS)}

\author{
W. Y. Yin, B. Guo, X. T. Dong, and Y. B. Gan \\ Temasek Laboratories \\ National University of Singapore \\ Singapore 119260
}

Received 17 March 2003

ABSTRACT: The effects of dielectric, radiative leakage and ohmic losses in CPWs on the propagation of Gaussian and sinusoidally modulated Gaussian pulses are investigated. These CPWs are assumed to be fabricated on a single-layer low-temperature co-fired ceramic (LTCC) substrate. It is shown that, based on the improved empirical formula for accurate prediction of the ohmic attenuation constant presented by Liao et al., the lossy effects in LTCC CPWs are dominated by the ohmic loss of the center and ground conductive planes in the frequency range up to $40 \mathrm{GHz}$. (C) 2003 Wiley Periodicals, Inc. Microwave Opt Technol Lett 39: 94-97, 2003; Published online in Wiley InterScience (www. interscience.wiley.com). DOI 10.1002/mop.11137

Key words: coplanar waveguides (CPWs); LTCCs; ohmic loss; pulse wave; attenuation

\section{INTRODUCTION}

The conductor losses of coplanar waveguides (CPWs) have been studied by many researchers using different techniques [1-9]. Physically, as the operating frequency increases, the dielectric loss, radiation loss, and ohmic loss of CPWs have significant effects on the pulse-waveform distortion $[7,8]$. In particular, Liao and Ponchak et al. have presented two sets of new empirical formulas to

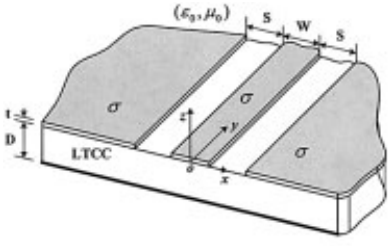

(a) Three-dimensional view

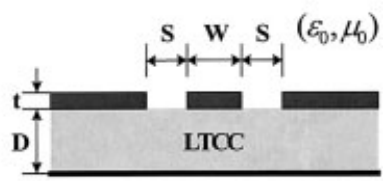

(b) Cross-sectional view
Figure 1 Geometry of an LTCC CPW: (a) three-dimensional view; (b) cross-sectional view

predict the ohmic loss for CPWs [8, 9], respectively, which are characterized by different conductivities and thicknesses of the center strip and ground planes over a wideband frequency range.

In this work, we focus on different lossy effects of lowtemperature co-fired ceramic (LTCC) CPWs on the transient characteristics of Gaussian or sinusoidally modulated Gaussian pulse propagation. Because LTCCs have excellent high-frequency performances, they have been widely used as substrates or superstrates in high-density interconnects and packaging systems [10, 11]. This paper is organized as follows: in section 2 , the geometry of a LTCC CPW is shown, and some formulas to determine the dielectric loss, radiation leakage loss and ohmic loss are introduced. Based on these formulas and the fast Fourier transformation (FFT), in section 3 , the transient propagated pulses are computed and compared, for different LTCC substrates and center strip and ground plane thicknesses, and their conductivities. Some conclusions of this work is presented in section 4.

\section{GEOMETRY}

The geometry of an LTCC CPW is shown in Figure 1, with LTCC thickness $D$, center strip width is $W$, and strip spacing $S$. Physically, the permittivity and loss tangent of LTCCs can be chosen as $\left(\varepsilon_{r}, \tan \delta\right)=(4.2,0.003),(5.9,0.002),(7.0,0.002),(7.8,0.0015)$, $(8.2,0.002)$, and $(10.6,0.001)$, respectively. The thickness and conductivity of the center strip and ground plane are characterized by $t$ and $\sigma$, respectively.

Mathematically, the attenuation constant that results from the dielectric loss of the LTCC substrate can be evaluated by [5]:

$$
\alpha_{d}=0.3145 \frac{\varepsilon_{r}}{\sqrt{\varepsilon_{r e}(f)}} \frac{\varepsilon_{r e}(f)-1}{\varepsilon_{r}-1} \frac{\tan \delta}{\lambda_{0}}(\mathrm{~Np} / \text { unit length }),
$$

where $\varepsilon_{r e}(f)$ is the effective dielectric constant of the CPW, and can be accurately determined by some empirical formulas; $f$ is the operating frequency, and $\lambda_{0}$ is the wavelength in free space. The attenuation constant of the CPW at high frequency due to the radiative leakage can be determined by $[5,8]$ :

$\alpha_{r}(f)=2\left(\frac{\pi}{2}\right)^{5}\left\{\frac{\left[1-\frac{\varepsilon_{r e}(f)}{\varepsilon_{r}}\right]^{2}}{\sqrt{\frac{\varepsilon_{r e}(f)}{\varepsilon_{r}}}}\right\} \frac{(w+2 s)^{2} \varepsilon_{r}^{3 / 2}}{c^{3} K^{\prime}(k) K(k)} f^{3}$

(Np/unit length),

where $k=w /(w+2 s)$, and $K(k)$ and $K^{\prime}(k)$ are the complete elliptic integrals of the first and second kinds, respectively. The attenuation constant of the CPW due to ohmic loss can be evaluated by [5]: 